\title{
Study of Working Medium on the Hydrophilic Surface of Evaporation Enhancement
}

\author{
Shen-Chun Wu, Sin-Jie Lin, Chun-Ko Lo and Wei-Jie Hsu \\ Dept. of Aviation Mechanical Engineering, China U of Science and Technology, Taipei, Taiwan.
}

\begin{abstract}
The main purpose of this paper is using modification of nanoparticle layers to increasing the hydrophilic surfaces and enhancing the evaporation behaviors in stainless steel plate. When the products being made, use three different kind of fluids, acetone, ethanol and water to testing the benefit for evaporation test. The experimental results showed that the unmodified stainless steel plate's contact angle 87 and the contact angle is changing to 7 by modified stainless steel plate of 18 layers. But use the best contact angle to testing for the evaporation test, it was found that the acetone evaporation was enhanced to $450 \%$ benefit, the ethanol evaporation was increased by about $350 \%$ benefit, and the evaporation which enhancing over $300 \%$ benefit was by water.
\end{abstract}

Keywords-nanoparticle layers; hydrophilic; surfaces modification; evaporation

\section{INTRODUCTION}

Hydrophilic surface is one of the important factor in evaporation and heat transfer performance. To enhance hydrophilic can increase the surface tension of liquid and the evaporation ratio is also faster. There are many efficient ways to improve the hydrophilic surface, nanoparticle layers with non-toxic, simple process and the advantages of visualization. Thetefore, this paper(article) chose nanoparticle layers as a surface modification to the method. So use nanoparticle layers method to improve the surface hydrophilic that can let the droplet's contact angle becomes small. Literature review follows: In 1934, Blodgett [1] first proposed and performed nanoparticle layers assembly on glass for hydrophobic surface modification. In 1966, Iler et al. [2] also used the nanoparticle layers assembly method to render the glass surface hydrophobic and unwettable. Choi et al.[3] The first time in 1995 on a metal surface, the use of nanoparticle layers to produce a hydrophilic surface, and found that the metal surface modification after the thermal conductivity is increased, but did not leave a results point of view. In 2006 Cebeci et al. [4] the use of nanoparticle layers method, the modified material on the glass surface to produce a hydrophilic surface, and make the best production process and found that the modified layer 14, the contact angle of less than $5^{\circ}$. In 2007, Bravo et al. [5] performed nanoscale bilayers assembly method for hydrophilic modification on glass surface and were able to decrease water's contact angle to $9^{\circ}$ using 15 layers. Phan [6] 2009 for the first time using nano lamination, different metal substrate surface modification on a stainless steel plate, also $\mathrm{SiO} 2$ nanoparticles laminated method, the modified layer 15 , the contact angle of $22^{\circ}$. Wu 2013a [7] Reference [5], [6] recommends that the more layers the contact angle of the smaller, and therefore the use of a modified stack technology, select the number of layers produced, modified (5.10.15) layer on the stainless steel plate, measured by the modified layer 15 contact angle $24^{\circ}$. Therefore, $\mathrm{Wu}$ et al.2013b [8] before extending the literature [7] approach, therefore making additional layers, found a better result in the contact angle of the modified layer 20 is reduced to $12^{\circ}$. Therefore, $\mathrm{Wu}$ et al. 2013c [9] looking for a smaller contact angle, continue to use the nanoparticle layers method, the modified layers is set (15.17.20.23.25) layer, found in the best layers 17 layer contact angle reduced to $11^{\circ}$, hydrophilic continue to improve, but also notice changes in the production of modified layers to further identify the relationship between the number of layers and the contact angle. And $\mathrm{Wu}[10]$ using nano technology to produce laminated stainless steel plate in 2015, published $(0-20)$ varies continuously modified layers, look for the results of more hydrophilic, simultaneously working medium is water evaporation tests found the number of layers in the layer 18 , the contact angle $7^{\circ}$. Using different working medium (ethanol, acetone, water) enhanced evaporation to make a surface modification of the substrate.

\section{NANOPARTIClE LAYERS ASSEMBLy FILM FOR MODIFICATION SURFACE}

Nanoparticle layers assembly making process is reference $\mathrm{Wu}[10]$, the substrate is stainless steel plate, size is $2 \mathrm{~cm} * 2 \mathrm{~cm}$ as shown in Figure 1. Saving time and cost and increasing the processed surface lifetime is all to avoid the product form damaging. Therefore, use multiple layers of fixation can let multi-piece stainless steel plates place in the holder(fixed mount) that is avoiding collisions and surface abrasion. The nano layer assembled film technology will be produced by three different functions of laminates in surface. As shown in Figure 1 comprising (A) adhesion layer, (B) body layer, (C) top layer, three parts. As known in figure 1, use 4 cups of liquor which have been soaked in the previous step. Then soak plating a layer of bilayer, and repeat stacking to complete layer-bylayer. The nanoparticle layers hydrophilic surface modification was divided into three parts, each part of production process and formula descriptions (Figure 1) as follows: A part is a production process of adhesive layer which describes by the previous step. This part needs to soak five times to complete the adhesive layer of production, and then establish the body layer, it will be better with body layered adsorption. 
Part B is the body layered process which describes by the previous step. This part is the key-layer part to change the contact angle, the best layer is 18 (layers).

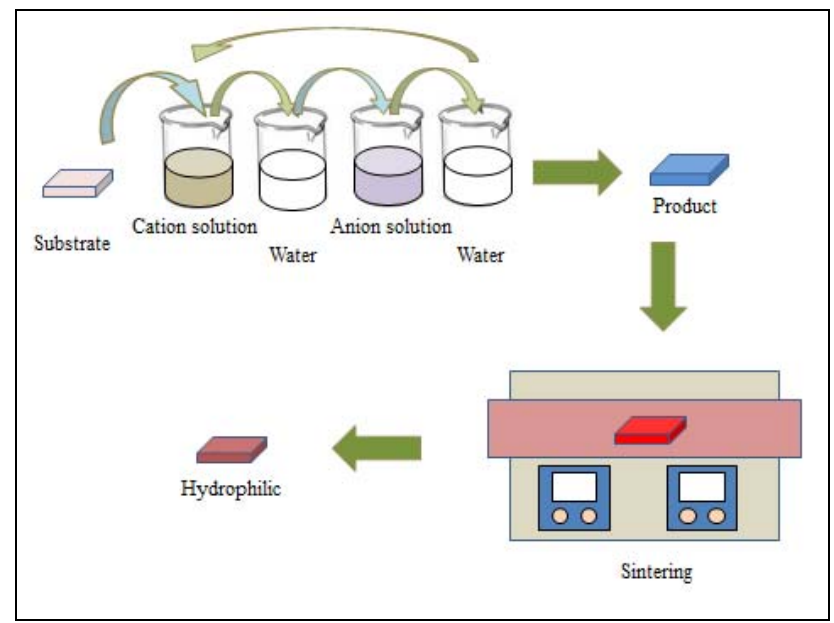

FIGURE I. PROCESS Of MANUFACTURE

Part $\mathrm{C}$ is top layer process which describes by the previous step. This layer is in order to protect nanoparticles attaching in stainless steel plate to prolong the life-span. Finish the top layer is completing the surface modification's pre-production. Then imbedding stainless Steel plate was plated nanoparticle film in the sintering furnace. Adjust the sintering parameters to sintering.

provided by the literature [10], setting $500^{\circ} \mathrm{C}$ and burning 4 hours. When the furnace is cooling, take it out. End of sintering, the hydroponic surface modification of stainless Steel plate is completed.

Sintering burn-off the unnecessary polymer and also let surface of Nano-layer to be more closer, more stronger and improve the intensity and life-span.

\section{EVAPORATION EFFICIENCY TEST}

(Figure 2) According to $\mathrm{Wu}[10]$ research, the best contact angle evaporation measurements and visualization diagram (Figure 3), and define the best modified layers is 18. In the visual observation experiment, put the unchanged tabletshaped(left) and modification of the best hydroponic flat(right) in the testing platform at the same time, then use the Pipette that can control the droplet size $(\mathrm{v}$, herein controlled $0.5 \sim 5 \mathrm{ul})$ dropping in the unmodified and modified tablet, next use the Top view's Camera to monitor the liquid film's (a) variable-area, add the time's record, and find out the evaporation area as time go on. In the experiment, the temperature and humidity will be control at the certain environment conditions. The humidity control at $50 \% \mathrm{RH}( \pm 8 \%)$, the temperature control at 25 degrees $\left( \pm 1{ }^{\circ} \mathrm{C}\right)$. In order to test the evaporation efficiency of different working medium, there are three different kinds of working medium such as ethanol, acetone and water to test the evaporation efficiency. The drop control in $0.5 \sim 5 \mathrm{ul}$ that accounts for the contact angle measurement which in $0.5 \sim 5 \mathrm{ul}$ can avoid effects of gravity to accuracy of the contact angle. Discovered in subsequent experiment, the larger droplet which has a little benefits in the evaporation experiment. In order to get the evaporation efficiency, therefore, use the formula (Equation 1) to calculate.

$$
\begin{aligned}
& \mathrm{t}^{*}=\frac{t_{\text {un mod }}-t_{\text {mod }}}{t_{\text {mod }}} \\
& \eta=\mathrm{t}^{*} \times 100 \%
\end{aligned}
$$

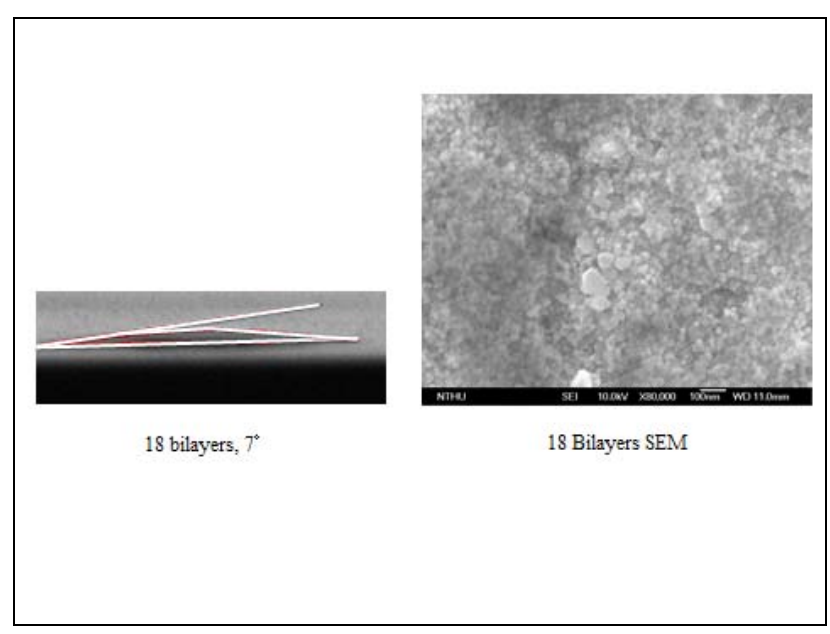

FIGURE II. ANGLE AND SEM

\section{RESUlTS AND DisCUSSION}

In this paper is successful use of the laminate assembly method, use the nanoparticle coating in stainless steel plate to produce a hydrophilic surface which contact angle is $7^{\circ}$ (Figure 2).

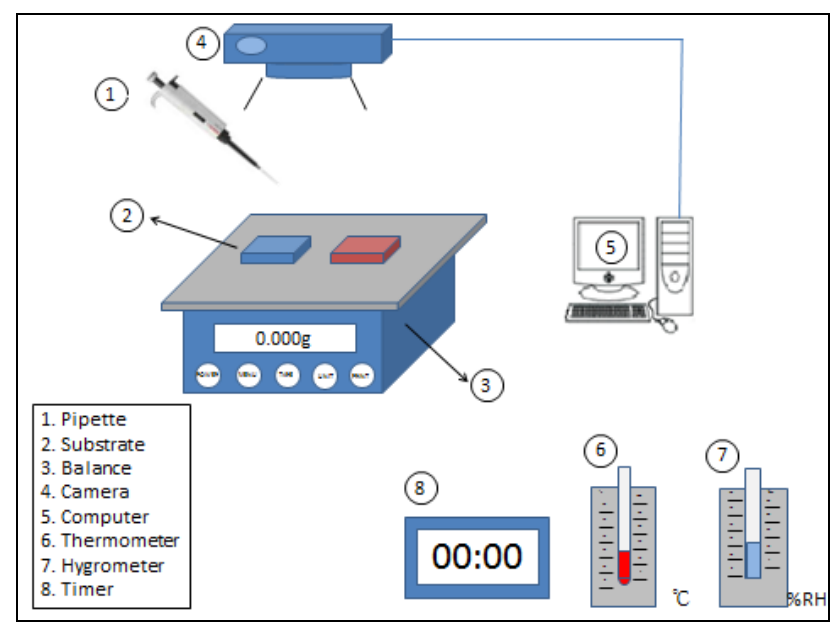

FIGURE III. SCHEMATIC EVAPORATION TEST

To get changed substance and unchanged substance's surface modification into the formula to compare their evaporation time (Equation 1), calculated that the evaporation ratio is $t^{*}$, then the $t^{*}$ multiply by $100 \%$ obtain the evaporation efficiency use the formula (Equation 2) to calculate. 
Measuring of $0.5 \mathrm{ul}$ of water in figure. 4 , that is $\mathrm{t}^{*} ; \mathrm{t}^{*}=2$, and lift $200 \%$, to make the evaporation efficiency as a basis for judgement. This paper will project the interaction between working medium (droplet size) and evaporation efficiency in figure 4 . The Figure includes the evaporation test results by ethanol, acetone and water, etc. The working medium is ethanol that evaporation efficiency is about $450 \%$. When the working medium is acetone, the evaporation efficiency is keeping about $400 \%$ on the increase by the test results as figure 4 shown. And the evaporation efficiency is also stable around $380 \%$ by water.

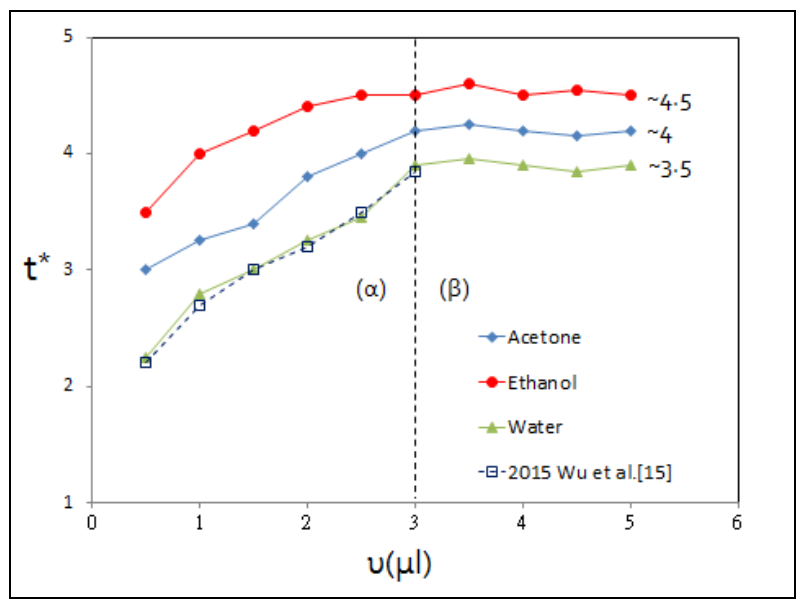

FIGURE IV. EVAPORATION EFFICIENCY OF DIFFERENT

According to the literature $\mathrm{Wu}[10]$, take water as the working medium to control in $0.5 \sim 3$ ul (Figure 4), but the evaporation efficiency is close to this paper's research. Comprehensive comparison of three working medium, the best is ethanol, second is acetone, and the last is water. This paper is just proving basis of the evaporation time. Depending on the different evaporation time that the result will be different. Based on speed of evaporation time, acetone $>$ ethanol $>$ water. Because of ethanol has lower surface tension and the best hydroponic, but the operations of temperature range and temperature change is of great benefit to acetone, so if just look at the evaporation time, acetone is most advantageous. But based on evaporation efficiency of modified, ethanol has a great benefit for enhancement.

\section{CONCLUSIONS}

This article aims is using nanoparticles assembled film laminated surface modification technology to increase the hydrophilic in stainless steel plate, then enhance the evaporation performance to research, conclusions of this paper are as follows:

\section{The contact angle results}

The successful production of a stainless steel plate surface $7^{\circ}$ minimal contact angle.

\section{Evaporation efficiency test results}

There is enhancing about $350 \%$ by water, enhancing about $400 \%$ by acetone, when the working medium is ethanol that is enhancing to $450 \%$ around. And the droplet is as smaller as faster in the evaporation time.

\section{ACKNOWLEDGEMENTS}

The authors would like to thank the ministry of science and technology of the Republic of China, Taiwan, for financially supporting this research under Contract Project:MOST1042221-E-157-008-.

\section{REFERENCES}

[1] Blodgett, K. B., "Monomolecular films of fatty acids on glass," Journal of the American chemical society, Vol.56, pp.495, 1934

[2] Iler, R. K., "Multilayers of colloidal particles," Journal of Colloid and Interface Science, Vol. 21, pp. 569, 1966.

[3] Choi, S.U.S., Eastman, J.A.,"Enhancing thermal conductivity of fluids with nanoparticles," American Mechanical Engineering Congress ,Vol. 231 ,pp. 99-105,1995.

[4] Cebeci, F. Cü ., Wu, Z., Zhai, L., Cohen, R. E., Rubner, M. F., "Nanoporosity-Driven Superhydrophilicity: A Means to Create Multifuncional Antifogging Coatings," Langmuir, Vol. 22, pp. 28562862, 2006.

[5] Bravo, J., Zhai, L., Wu, Z., Cohen R. E., and Rubner, M. F., "Transparent Superhydrophobic Films Based on Silica Nanoparticles," Langmuir, Vol. 23, pp. 7293-7298, 2007.

[6] Phan, H.T., Caney, N., Marty, P., Colasson, S., Gavillet, J., "Surface wettability controlled by nanocoating: the effects on pool boiling heat transfer and nucleation mechanism," International Journal Heat and Mass transfer, Vol. 52, pp. 5459-5471, 2009.

[7] Wu, S.C., Hsu, C.T., Yang, W.H., Wang,D., Li, H. S., Huang, Z. Y., Su, S. J., and Chen, Y. M. "Investigating the application of nanoscale bilayers assembly on stainless steel plate to evaporation behavior, "Advanced Materials Research , Vol. 750, pp.2104-2107, 2013a.

[8] Wu, S.C. , Wang, D. , Li, H. S. , Lin, S. J. , Hsu, C. T. , and Chen , Y. M. ," Investigating the use of nanoscale bilayers assembly on stainless steel plate to improve evaporation," Applied Mechanics and Materials , Vol. 395-396, pp. 718-725,2013b.

[9] Wu, S.C. , Wang, D. , Lin , S. J., Ciou, P .W. , Chung, C.Y., Su, S.J. , and Chen, Y.M. "' Investigating the use of nanoscale bilayers assembly on stainless steel plate for surface hydrophilic modification, " Applied Mechanics and Materials, Vols. 401-403, pp. 792-795,2013c.

[10] Wu, S.C. , Wang, D. , Lin , S. J. , and Chen , Y.M. , "Investigating the Effect of Hydrophilic Surface Modification on Droplet Evaporation ," Advanced Materials Research, Vols. 1120-1121, pp. 779-784,2015. 